\title{
Geometria proiettivo-differenziale di alcune varietà equi-parametriche.
}

\author{
Memoria di Inte Popa e Gheorghe Gheorghiev (a Iasi) \\ A. Enrico Bompiani in occasione del suo Giubileo scientifico.
}

\begin{abstract}
Sunto - Sono studiate, dal punto di vista proiettivo, alcune variètà complesse generate da elementi (punti, rette o piani) appartenenti ad un tetraedro e dipendenti da un solo parametro. Si mettono in luce $i$ legami (subordinazioni) tra le geometrie di tali varietà.
\end{abstract}

1. Nella geometria proiettiva, sono studiate di solito configurazioni generate da punti, rette o piani. Un punto di $S_{3}$ genera-secondo il numero dei parametri di quali esso dipende-una linea, una superficie oppure un dominio spaziale con una certa organizzazione di suoi punti; una retta genera nna superficie rigata, una congruenza, un complesso oppure la totalità delle rette organizzata in un certo modo; un piano genera configu. razioni duali alle prime.

Considerando, come elemento generatore, combinazioni di punti, rette o piani, si ottengono generalmente varietà non olonome.

In ciò che segue, saranno considerate varietà generate da elementi appartenenti ad un tetraedro. $\grave{E}$ utile osservare che la geometria di alcune configurazioni è subordinata a quella delle altre; criterio di subordinazione essendo la povertà di componenti nell' elemento generatore. Infatti, se la varietà $v$ e $V$ hanno elementi generatori $e$, rispettivamente, $E$ con $e \subset E$, è sempre possibile colmare la differenza $E-e$ per la considerazione di opportuni intorni di $e$ su $v$, cioè ridurre la geometria di $v$ a quella di $V$; gli invarianti di $v$ si otterranno per certe particolarizzazioni di quelli di $V$. La geometria di qualunque varietà è subordinata a quella della varietà generata dal quadripunto $A_{0} A_{1} A_{2} A_{3}$; per questa, le formule di FrENETCARTAN

$$
d A_{i}=\omega_{i}^{j} A_{j}
$$

contengono tutte le proprietà. 
Si può dunque compiere la geometria di uma varietà $v$ in due tappe:

1) Subordinare la geometria di $v$ a quella, gia costruita, di un'altra varietà $V$ e

2) Colmare la differenza $E-e$ coll'introduzione di opportuni intorni di $e$ su $v$.

In questo modo, le interpretazioni geometriche del riferimento e degli invarianti di $v$ sono semplici conseguenze di quelle relative a $V$.

Se le componenti punti, rette o piani dell' elemento generatore dipendono dallo stesso numero di parametri, diremo che la varietà $\dot{e}$ equi-parametrica. La geometria di tale varietà può essere considerata, nello stesso tempo, come quella della corrispondenza tra configurazioni descritte dalle singole componenti.

2. Per illustrare le generalità precedenti, consideriamo le varietà generate da:

$E_{3}$ : due rette non incidenti

$E_{2}: \gg \gg$ incidenti

$E_{3}: \gg$ punti

$E_{4}$ : un piano, una retta nel piano e un punto su di essa

$E_{5}: \gg \gg \theta$ un punto incidenti

$E_{6}:$ punto e una retta per esso

$E_{7}$ : una retta

$E_{8}$ : un punto.

Si vede subito che hanno luogo le inclusioni:

$$
\begin{aligned}
& E_{1} \supset E_{7} ; \\
& E_{2} \supset E_{4} ; E_{4} \supset E_{5} \supset E_{8} ; E_{4} \supset E_{6} \supset E_{7} ; \\
& E_{3} \supset E_{8} ; E_{6} \supset E_{8} .
\end{aligned}
$$

Notiamo che $E_{2}$ non è incluso in $E_{1}$, ma è un caso d'eccezione.

Per esempio, l'inclusione $E_{2} \supset E_{5}$ mostra che la geometria di una varietà non olonome lineare $V_{3}^{2}$ può essere dedotta da quella della coppia di varietà $V_{3}^{1}$ a centri coincidenti; l'inclusione $L_{2} \supset E_{6}$ ci mostra che la geometria della varietà di coni lo studio della quale è stato schizzato da noi recentemente $[I]$ è riducibile a quella di due varietà di coni associate. 
Appaiono come irriducibili nel gruppo di varietà considerate la geometria delle varietà generate da $E_{1}, E_{2}, E_{3}$, lo studio delle quale dev'essere fatto direttamente.

In ciò che segue, ci limiteremo alle varietà uniparametriche. Fra poco, presenteremo lo studio di qualche altre varietà.

3. Abbiamo mostrato altrove [2], [3] che la geometria della varietà uni-parametrica generata da $E_{2}$ è governata dalle formule di FRENET

$$
\begin{aligned}
\frac{d A_{0}}{d s}=\dot{A}_{0} & =\tau_{1} A_{0}+A_{3} \\
\dot{A}_{1} & =A_{0}+\left(x_{1}-\tau_{1}\right) A_{1}+A_{2} \\
\dot{A}_{2} & =x_{2} A_{0}+A_{1}-\tau_{1} A_{2} \\
\dot{A}_{3} & =\tau_{2} A_{0}+x_{3} A_{1}+x_{4} A_{2}+\left(\tau_{1}-x_{1}\right) A_{3},
\end{aligned}
$$

dove $d s=\omega_{0}^{3}$ è l'invariante differenziale del prim'ordine, $\chi_{1} \ldots \chi_{4}$ invarianti

\begin{tabular}{|c|c|c|c|c|c|c|}
\hline$\gg \gg$ & $\gg$ & 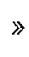 & $\gg$ & $E_{5}$, & $»$ & $x_{2}=x_{4}=0$ \\
\hline$»$ & $\gg$ & $»$ & $»$ & $E_{8}$ & $»$ & $x_{2}=x_{4}=x_{1}=x_{3}-2 x_{3} \tau_{1}=0$ \\
\hline$\gg$ & $»$ & » & $»$ & $E_{6}$ & $»$ & $x_{2}=\tau_{1}=0$ \\
\hline$\gg$ & $»$ & » & $»$ & $E_{7}$, & $»$ & $x_{2} \equiv \tau_{1}=\dot{x}_{4}-2 x_{1} x_{4}+2 x_{3}=0$ \\
\hline
\end{tabular}
finiti dal secondo ordine $e \tau_{1}, \tau_{2}$ del terzo ordine della varietà.

Si ottengono le geometrie delle varietà subordinate:

per la varietà generata da $E_{4}$, prendendo $x_{2}=0$

Notiamo che, per questa via, le geometrie delle curve sghembe, $E_{\mathbf{8}}$ elemento generatore, delle superficie rigate $E_{7}$, delle bande rigate $E_{6}$ e degli elementi di contatto $E_{5}$ si deducono da quella di due superficie rigate con intersezione non lineare. Da un altro canto, è da notare che la geometria di quest' ultima configurazione $E_{2}$ elemento generatore è semplicissima appunto per il fatto che il riferimento d'ordine zero è più ampio degli altrí.

4. Coppie di superficie rigate elemento generatore $E_{1}$.

Nel 1923, E.P. LaNe ha abbozzato lo studio della corrispondenza tra due superficie rigate [4]; I. Creanga [5] e, più recentemente, E. T. IvLEV [6] sono tornati sullo stesso argomento.

Siano $\mathrm{R}, \mathrm{R}^{*}$ le superficie rigate generate da $g=A_{0} A_{1}, g^{*}=A_{2} A_{3}$. Per 
i riferimenti d'ordine zero, avremo

$$
\pi_{0}^{2}=\pi_{0}^{3}=\pi_{1}^{2}=\pi_{1}^{3}=\pi_{2}^{0}=\pi_{2}^{1}=\pi_{3}^{0}=\pi_{3}^{1}=0 .
$$

Supposto $\omega_{0}^{3} \neq 0$, l'annullarsi di $\omega_{0}^{3}$ dipende soltanto dalla scelta del riferimento, si avrà

$$
\begin{gathered}
\omega_{0}^{2}+a \omega_{0}^{3}=\omega_{1}^{2}+b \omega_{0}^{3}=\omega_{1}^{3}+c \omega_{0}^{3}=\omega_{k}^{0}+e \omega_{0}^{3}=\omega_{2}^{1}+ \\
+f \omega_{0}^{3}=\omega_{3}^{0}+h \omega_{0}^{3}=\omega_{3}^{1}+k \omega_{0}^{3}=0 .
\end{gathered}
$$

Si può scegliere

$$
a=c=e=k=0, \quad b=h=-1 .
$$

Le generatrici $g+d g, g^{*}+d g^{*}$ hanno - in coordinate locali - le equazioni :

$$
\begin{array}{ll}
x^{2}=\omega_{0}^{2} x^{0}+\omega_{1}^{2} x^{1} & x^{0}=\omega_{2}^{0} x^{2}+\omega_{3}^{0} x^{3} \\
x^{3}=\omega_{0}^{3} x^{0}+\omega_{1}^{3} x^{1} & \text { rispettivamente } \\
x^{1}=\omega_{2}^{1} x^{2}+\omega_{3}^{1} x^{3}
\end{array}
$$

ciò che significa che $A_{0} A_{3}, A_{1} A_{2}$ sono le rette che incontrano la quadrupla $g, g^{*}, g+d g, g^{*}+d g^{*}$ e che i casi esclusi $b$ o $h=0$ con $a=c=e=k=0$ significano che $R$ e $R^{*}$ sono sviluppabile

La scelta fatta hil come conseguenza :

$$
\pi_{0}^{1}=\pi_{1}^{0}=\pi_{2}^{3}=\pi_{3}^{2}=0, \quad \pi_{0}^{0}=\pi_{\varepsilon}^{3}, \pi_{1}^{1}=\pi_{2}^{2}
$$

dalla quale risulta, in primo luogo:

$$
\begin{gathered}
\omega_{0}^{1}+A \omega_{0}^{3}=\omega_{1}^{0}+B \omega_{0}^{3}=\omega_{2}^{3}+C \omega_{0}^{3}=\omega_{3}^{2}+E \omega_{0}^{3}=\omega_{0}^{0}-\omega_{3}^{3}+ \\
+F \omega_{0}^{3}=\omega_{1}^{1}-\omega_{2}^{2}+G \omega_{0}^{3}=0
\end{gathered}
$$

e, in secondo, che $\omega_{0}^{3} \cdot$ e $f \neq-1\left({ }^{1}\right)$ sono invarianti del primo ordine della varietà. L' invariante $f$ sarà notato per $-x$. L'interpretazione di $\omega_{0}^{3}$ e $x$ è semplicissima: i birapporti delle quaterne determinate da $g, g+d g, g^{*}$ $g^{*}+d g^{*}$ su $A_{0} A_{3}, A_{1} A_{2}$ sono $\left(\omega_{0}^{3}\right)^{2}$, rispettivamente, $x\left(\omega_{0}^{3}\right)^{2}$. In legame con lo stesso intorno del primo ordine della varietà, si può osservare:

Sia $P(\lambda, \mu, 0,0)$ un pnnto di $g$; il piano tangente in $P$ a $R$ sega $g^{*}$ in $P^{*}(0,0, \mu, \lambda)$ ciò che vuol dire che la corrispondenza tra $R$ e $R^{*}$ induce

(1) Il caso escluso, $f=-1$, sarà studiato separatamente. 
un'omografia tra le punteggiate $g, g^{*}$. Il piano tangente in $P^{*}$ a $R^{*}$ sega $g^{\prime}$ in $P_{1}(\lambda, \mu x, 0,0)$. Si ottiene così la serie di punti, su $g, P_{n}\left(\lambda, \mu x^{n}, 0,0\right)$ convergenti ai vertici $A_{0}$ oppure $A_{1}$ secondo che $|x|<1$ oppure $|x|>1$.

Nelle relazioni precedenti, si può prendere $B=-1$ e si avrà $\pi_{0}^{0}=\pi_{1}^{1}$. Come $\Sigma \pi_{i}^{i}=0$, ne segue che tutti $i$ parametri secondari $\pi_{j}^{i}$ sono nulli dunque che il riferimento canonico è del secondo ordine; i coefficienti $A, C, E, F, G$ sono invarianti, pare del secondo ordine, della varietà; in fine, da $\pi_{0}^{0}=0$, risulta che $\omega_{0}^{0}=\rho \omega_{0}^{3}$ con $\rho$ invariante del terzo ordine della varietà. In conelusione, la varietà $R R^{*}$ ha un invariante differenziale $\left(\omega_{0}^{3}\right)$ e uno finito $x$ del primo ordine, 5 invarianti del secondo ordine che saranno notati per $\tau_{1}, \ldots, \tau_{5}$ e uno del terzo ordine $p$. Le formule di FreneT-CartaN sono

$$
\begin{aligned}
\frac{d A_{0}}{\omega_{0}^{3}}=\dot{A}_{0} & =\rho A_{0}+\tau_{1} A_{1}+A_{3} \\
\dot{A}_{1} & =A_{0}-\left(\rho+\tau_{4}+\tau_{5}\right) A_{1}+A_{2} \\
\dot{A}_{0} & =x A_{1}+\left(\tau_{4}-\rho\right) A_{2}+\tau_{2} A_{3} \\
\dot{A}_{3} & =A_{0}+\tau_{3} A_{2}+\left(\rho+\tau_{5}\right) A_{3}
\end{aligned}
$$

e le condizioni d'immobilità

$$
\begin{aligned}
& \frac{\dot{x}^{0}+p x^{0}+x^{1}+x^{3}}{x^{0}}=\frac{\dot{x}^{1}+\tau_{1} x^{0}-\left(\rho+\tau_{4}+\tau_{5}\right) x^{2}+x x^{2}}{x^{1}}= \\
& \frac{\dot{x}^{2}+x^{1}+\left(\tau_{4}-\rho\right) x^{2}+\tau_{8} x^{3}}{x^{2}}=\frac{\dot{x}^{3}+x^{0}+\tau_{2} x^{2}+\left(\rho+\tau_{5}\right) x^{3}}{x^{3}} .
\end{aligned}
$$

Interpretazioni geometriche.

In ciò che riguarda il riferimento, rimane a caratterizzare il punto unitario. Consideriamo, in primo luogo, la retta caratteristica del piano $A_{1} A_{2} A_{3}\left(x^{0}=0\right)$. Conformemente alle condizioni d'immobilità, le sue equazioni sono

$$
x^{0}=x^{1}+x^{3}=0 .
$$

La sua intersezione con $A_{1} A_{3}$ è il punto $V_{13}(0,1,0,-1)$ il oui armonico rispetto alla coppia $A_{1}, A_{3}$ è il punto unitario $U_{13}$ sullo spigolo $A_{1} A_{3}$.

Il piano $A_{0} A_{2} U_{13}$ ha l'equazione

$$
x^{1}-x^{3}=0
$$


In secondo luogo, la tangente in $A_{1}$ alla curva descritta da questo punto ha le equazioni :

$$
x^{3}=x^{0}-x^{2}=0
$$

Il piano definito da $U_{13}$ e questa tangente è $x^{0}-x^{2}=0$.

In fine, la quadrica pre-oseulatrice a $R$ lungo $g$ contenente la generatrice $g^{*}$ ha l'equazione

$$
x^{0} x^{2}-x^{1} x^{3}=0
$$

Questa quadrica e i due piani considerati cui sopra hanno in comune due pnnti, uno di essi essendo il punto unitario cercato.

Interpretazioni geometriche degli invarianti $\tau_{1}, \ldots, \tau_{5} e$.

La tangente in $A_{0}$ alla curva descritta da questo punto ha le equazioni:

$$
x^{2}=x^{1}-\tau_{1} x^{3}=0 .
$$

Ne segue l'interpretazione di $\tau_{1}$ come un birapporto. Nello stesso modo, le tangenti in $A_{2}$ e $A_{3}$, ai luoghi di questi punti, essendo

$$
x^{0}=x x^{3}-\tau_{2} x^{1}=0, \quad \text { rispettivamente, } \quad x^{1}=x^{2}-\tau_{3} x^{3}=0
$$

si ottengono interpretazioni per $\tau_{2}, \tau_{3}$.

Le quadriche osculatrici a $R$ o $R^{*}$ lungo $g$, rispettivamente, $g^{*}$, sono

$$
\begin{gathered}
2 x^{0} x^{2}-2 x^{1} x^{3}+\left(\tau_{2}-1\right)\left(x^{2}\right)^{2}-2 \tau_{4} x^{2} x^{3}+\left(\tau_{1}-\tau_{3}\right)\left(x^{3}\right)^{2}=0 \\
2 x x^{0} x^{2}-2 x^{1} x^{3}+\frac{\tau_{2}-x}{x}\left(x^{1}\right)^{2}-\left(2 \tau_{4}-\frac{\dot{x}}{x}\right) x^{0} x^{1}+\left(\tau_{1}-x \tau_{3}\right)\left(x^{0}\right)^{2}=0
\end{gathered}
$$

il piano polare di $A_{2}$ rispetto $Q$ ha l'equazione

$$
x^{0}-\tau_{4} x^{3}=0
$$

che fornisce 1'interpretazione di $\tau_{4}$.

I piani

$$
x^{1}-x^{2}=0, x^{0}-x^{2}=0
$$

passanti per spigoli e punto unitario del riferimento, hanno significato 
geometrico; le loro rette caratteristiche

$$
x^{1}-x^{2}=\tau_{1} x^{0}-\tau_{3} x^{3}+\left(1-x+2 \tau_{4}+\tau_{5}\right) x^{1}=0
$$

rispettivamente

$$
x^{0}-x^{2}=\left(2 \rho-\tau_{4}\right) x^{0}+\left(\tau_{3}+1\right) x^{0}=0
$$

forniscono-se $\tau_{1}, \tau_{3}$ non sono simultaneamente nulli, rispettivamente $\tau_{3} \neq-1$ interpretazioni per $\tau_{5}$ e $\rho$.

Altre interpretazioni sono fornite dai piani osculatori alle curve descritte dai vertici del riferimento, oppure dai punti caratteristici delle sue faccie.

Nello stesso scopo, si potrebbero considerare altri enti geometrici collegati alla configurazione; per esempio, i complessi lineari aventi contatto di second'ordine con una delle rigate e del primo coll'altra:

$$
\left.\left(\tau_{3}-\tau_{1}\right) p_{03}+\tau_{2}-1\right) p_{12}=0
$$

rispettivamente

$$
\left(x \tau_{3}-\tau_{1}\right) p_{03}+\left(\tau_{2}-\chi\right) p_{12}=0
$$

Coppie reciproche.

Le rette $\bar{g}=A_{0} A_{3}, \bar{g}^{*}=A_{1} A_{2}$ generano le superficie rigate $\bar{R}, \bar{R}^{*}$.

Le rette $\bar{g}+\overline{d g}, \bar{g}^{*}+\overline{d g}^{*}$ infinitamente vicine a $\bar{g}, \bar{g}^{*}$ hanno le equa. zioni

$$
\begin{array}{ll}
x^{1}=\tau_{1} \omega_{0}^{3} x^{0} & x^{0}=\omega_{0}^{3} x^{1} \\
x^{2}=\tau_{2} \omega_{0}^{3} x^{3} & \text { rispettivamente, }, \\
& x^{3}=\tau_{2} \omega_{0}^{3} x^{2}
\end{array}
$$

ne segue che le rette appoggiantesi su $\bar{g}, \bar{g}^{*}, \bar{g}+d \bar{g}, \bar{g}^{*}+d \bar{g}^{*}$ sono $g$ e $g^{*}$. La coppia $R, R^{*}$ sarà perciò chiamata reciproca della coppia $\bar{R}, \bar{R}^{*}$. Escludendo i casi $\tau_{1} \tau_{2} \tau_{3}=0-$ quando $\bar{R}$ o $\bar{R}^{*}$ sarebbe sviluppabile - birapporti delle quaterne determinate da $\bar{g}, \bar{g}^{*}, \bar{g}+d \bar{g}, \bar{g}^{*}+\bar{g}^{*}$ su $g$ e $g^{*}$ hanno $\mathrm{i}$ valori $\tau_{1}\left(\omega_{0}^{3}\right)^{2}, \tau_{2} \tau_{3}\left(\omega_{0}^{3}\right)^{2}$. La geometria della coppia reciproca è riducibile a quella della coppia iniziale scambiando l'invariante differenziale $\omega_{0}^{3}$ per $\sqrt{\tau_{1}} \omega_{0}^{3}$ oppure per $\sqrt{\tau_{2} \tau_{3}} \omega_{0}^{3}$. L'invariante $\bar{x}$ sarà $\frac{\tau_{1}}{\tau_{2} \tau_{3}}$ oppure l'inverso di questo.

\section{Corrispondenze notevoli.}

Da ciò che precede, segue che la corrispondeza con $\tau_{1}=\tau_{2} \tau_{3}$ è tale che la coppia reciproca sia eccezionale $\bar{x}=1$. Questa corrispondenza (ma per la 
quale $\left.x \neq 1, \quad \tau_{1}-\tau_{3} \neq 0, \quad \tau_{2}-1 \neq 0, \tau_{1}-x \tau_{3} \neq 0, \tau_{2}-x \neq 0\right)$ è caratterizzata anche dal fatto che i complessi lineari $C, C^{*}$ coincidono cioè che gli intorni di second'ordine delle rigate $R$ e $R^{*}$ appartengono allo stesso complesso lineare.

I casi più particolari $\tau_{1}-\tau_{3}=\tau_{2}-1=0$ oppure $\tau_{1}-x \tau_{3}=\tau_{2}-x=0$ sono caratterizzati dal fatto che uno o l'atro dei detti complessi $C, C^{*}$ sono indeterminati.

In fine, casi più particolari sono quelli per i quali

$$
\tau_{1}-\tau_{3}=\tau_{2}-1=\tau_{4}=0 \text { oppure } \tau_{1}-x \tau_{3}=\tau_{2}-x=\tau_{4}-\frac{\dot{x}}{2 x}=0
$$

per $i$ quali la quadrica osculatrice ad una delle rigate passa per la generatrice dell' altra.

Tornando alla corrispondenza indotta tra le punteggiate $g$ e $g^{*}$ da quella tra le rigate $R$ e $R^{*}$, si può chiedere quando la tangente in un punto $P(1, t, 0,0)$, alla curva da esso descritta, incontra $g^{*}$ e quella nel punto corrispondente $P^{*}(0,0, t, 1)$ incontra $g$.

La tangente in $P$ ha le equazioni

$$
\begin{gathered}
x^{1}-t x^{0}=S x^{3} \\
x^{2}=t x^{3}
\end{gathered}
$$

$\operatorname{con} S=\dot{t}-t^{2}-t\left(2 \rho+\tau_{4}+\tau_{5}\right)+\tau_{1}$

la tangente in $P^{*}$ ha le equazioni

$$
\begin{gathered}
x^{2}-t x^{3}=T x^{0} \\
x^{1}=t x x^{0}
\end{gathered}
$$

con $T=\dot{t}-\tau_{2} t^{2}-\left(2 \rho-\tau_{4}+\tau_{5}\right) t+\tau_{3}$.

Le condizioni imposte mostrano che le curve descritte da $P, P^{*}$ appar. tengono alle famiglie " $R$ ": $S=0, T=0$. Queste famiglie coincidono se e solo se

$$
\tau_{3}-\tau_{1}=\tau_{2}-1=\tau_{4}=0
$$

\section{Osservazione.}

Dallo studio della corrispondenza tra le superficie rigate $R, R^{*}$, si potrebbe dedurre quello del complesso composto dalle $\infty^{1}$ congruenze lineari aventi come direttrici la coppia $g, g^{*}$. Tali complessi sono stati studiati da diversi geometri, tra i quali Mentré il quale ne ha determinato la gene. ralità e ha mostrato diversi modi di generare tali complessi per complessi lineari [7]. 


\section{Il caso eccezionale.}

Nelle considerazioni precedenti, abbiamo escluso il caso $\omega_{2}^{1}=\omega_{0}^{3}$, essendo soddisfatte le relazioni $\omega_{0}^{2}=\omega_{2}^{0}=\omega_{1}^{3}=\omega_{3}^{1}=0$ e $\omega_{1}^{2}=\omega_{3}^{0}=\omega_{0}^{3}$.

Si può vedere subito che anche nel caso $\omega_{2}^{1}=\omega_{0}^{3}$ accanto alle relazioni precedenti si ha $d \omega_{0}^{3} \equiv 0$ dunque che $\omega_{0}^{3}$ è invariante differenziale della varietà .

Le consegnenze differenziali di questo sistema sono

$$
\omega_{2}^{3}=\omega_{1}^{0}+A \omega_{0}^{3}, \quad \omega_{3}^{2}=\omega_{0}^{1}+B \omega_{0}^{3}, \quad \omega_{3}^{3}=\omega_{0}^{0}+C \omega_{0}^{3}, \quad \omega_{2}^{2}=\omega_{1}^{1}+D \omega_{0}^{3} .
$$

Scegliendo $A=B=0 \quad C \neq D$, si avrà - dalle prime due relazioni -

$$
\omega_{1}^{0}=\alpha \omega_{0}^{3}, \quad \omega_{0}^{1}=\tau \omega_{0}^{3}
$$

e - dalle seconde -

$$
\omega_{2}^{2}=\omega_{1}^{1}+x_{1} \omega_{0}^{3}, \quad \omega_{3}^{3}=\omega_{0}^{0}+\chi_{2} \omega_{0}^{3}
$$

con $x_{1}, x_{2} \tau$ invarianti.

Si può prendere $\alpha=1$, con la quale tutti $\pi_{i}^{i}$ risultano nulli $e$, come conseguenza differenziale, $\omega_{0}^{0}=p \omega_{0}^{3}$ con $\rho$ pure invariante.

Con ciò, le formole di Frente-OARTAN sono

$$
\begin{aligned}
& A_{0}=\rho A_{0}+\tau A_{1}+A_{3} \\
& \dot{A}_{1}=A_{0}-\left(\rho+\frac{x_{1}+x_{2}}{2}\right) A_{1}+A_{2} \\
& \dot{A_{2}}=A_{1}+\left(\frac{x_{1}-x_{2}}{2}-\rho\right) A_{2}+A_{3} \\
& \dot{A_{3}}=A_{0}+\tau A_{2}+\left(\rho+x_{2}\right) A_{3} .
\end{aligned}
$$

Notiamo che queste formule sono del tutto simili a quelle del caso non eccezionale più precisamente: 1 nella vece di $x_{1} ; \tau_{1}=\tau_{3}=\tau ; \tau_{2}=1$; $\tau_{4}=\frac{x_{1}-x_{2}}{2} ; \tau_{5}=x_{2}$ ciò che ci dispensa d'interpretare questi invarianti.

Tale corrispondenza gode di notevoli proprietà.

La più semplice è quella che le omografie da essa indotte sulle punteg. giate $g$ e $g^{*}$ sono delle identità.

Un'altra è quella che gli intorni di prim'ordine delle due rigate $R, R^{*}$ appartengono alla stessa quadrica. Ne segue che $i$ complessi lineari a contatto rigato del prim' ordine con una delle rigate e di ordine zero con l'altra ha contatto del prim' ordine anche con questa. Ma di più, gli intorni 
del terz' ordine delle due rigate appartengono allo stesso complesso lineare di equazione $p_{12}-\tau p_{03}=0$ che è speciale se $\tau=0$. Questa proprietà d̀ caratteristica per il caso presente.

Se, di più, tra gli invarianti della corrispondenza ha luogo la relazione $\dot{\tau}=2 \tau\left(\rho+x_{2}\right)$, questo complesso è fisso e le rigate in corrispondenza appartengono ad un complesso lineare. Si può dire che questa è la più generale corrispondenza tra le rigate di un complesso lineare. Per il cambiamento del punto unitario

$$
A_{0}=\sqrt{\tau} \overline{B_{0}}, A_{1}=\frac{1}{\sqrt{\tau}} B_{1}, A_{2}=\frac{1}{\sqrt{\tau}} B_{2}, A_{3}=\sqrt{\tau} B_{\text {s }}
$$

si ottengono le formule semplicissime di tale corrispondenza

$$
\begin{array}{ll}
\dot{B}_{0}=-x_{2} B_{0}+B_{1}+B_{3} & B_{2}=B_{1}+\frac{x_{1}+x_{2}}{2} \dot{B}_{2}+\tau B_{3} \\
\dot{B}_{1}=\tau B_{0}+\frac{x_{1}-x_{2}}{2} B_{1}+B_{2} & \dot{B}_{3}=B_{0}+B_{2} .
\end{array}
$$

Un'altra proprietà caratteristica del caso eccezionale è la seguente:

La corrispondenza considerata stabilisce una trasformazione asintotica tra le rigate $R$ e $R^{*}$.

Da una parte, si corrispondono le asintotiche rettilinee; da nn'altra, le asintotiche eurvilineare nei punti corrispondenti $\left.P(1, t, 0,0), P^{*} 0,0, t, 1\right)$, - per l'omografia indotta tra le punteggiate $g, g^{*}$ - si ottengono dalla stessa equazione

$$
\dot{t}-t^{2}-\left(2 \rho+x_{2}\right) t+\tau=0 .
$$

Reciprocamente, nel caso della corrispondenza non-ecezionale, le asintotiche in $P$ e $P^{*}$ hanno le equazioni

$$
2 \dot{t}-t^{2}\left(\tau_{2}+1\right)-2 t\left(2 \rho+\tau_{5}\right)+\tau_{1}+\tau_{3}=0
$$

rispettivamente

$$
2 \dot{t}-t^{2}\left(\tau_{2}+x\right)-2 t\left(2 \rho+\tau_{5}-\right) \frac{\dot{x}}{2 x}+\frac{\tau_{1}}{x}+\tau_{3}=0
$$

esse coincidono se, e solo se, $x=1$.

5. Coppie di curve. - Lo studio di tale configurazione ha fatto l'oggetto di moltissimi lavori specialmente da quelli di Halphen, Berzolari, BomPIANI ecc.; più recentemente, lo studio delle coppie di curve è stato ripreso da M. Barner in un lavoro [8] di cui abbiamo notizie da Mathematical Reviews. 
Siano $\Gamma_{0}$ e $\Gamma_{1}$ due curve sghembe del ' $S_{3}$ proiettivo; $A_{0}$ e $A_{1}$ punti generici su di esse. Esprimendo che, nei riferimenti d'ordine zero, $A_{0}$ e $A_{1}$ sono punti fissi, arremo $\pi_{0}^{i}=\pi_{0}^{2}=\pi_{0}^{3}=0, \pi_{i}^{0}=\pi_{1}^{2}=\pi_{1}^{3}=0$. Si può conside. rare $\omega_{0}^{3} \equiv \equiv 0$ (il caso escluso, $\omega_{0}^{3} \equiv 0$, si riferisce soltanto alla scelta del riferimento). Si avrà dunque

$$
\omega_{0}^{1}+\alpha \omega_{0}^{3}=\omega_{0}^{2}+b \omega_{0}^{3}=0, \quad \omega_{1}^{0}+c \omega_{0}^{3}=\omega_{1}^{2}+f \omega_{0}^{0}=\omega_{1}^{3}+g \omega_{0}^{3}=0 .
$$

Possiamo prendere $a=b=0, c=g=0, f=-1$. Ne segue: $\pi_{3}^{1}=\pi_{3}^{2}=0$, $\pi_{2}^{3}=\pi_{2}^{0}=0, \pi_{0}^{0}+\pi_{z}^{2}=0$. Questa scelta significa semplicemente che lo spigolo $A_{0} A_{3}$ è la tangente in $A_{0}$ a $\Gamma_{0}$ e $A_{1} A_{2}$ è quella a $\Gamma_{1}$ in $A_{1}$. Il caso escluso, $f=0$ accanto a $b=0$ significa che le tangenti a $\Gamma_{0}$ e $\Gamma_{1}$ nei punti $A_{0}, A_{1}$ s'incontrano. C'è, d'una parte, un'ampia generalizzazione proiettiva della trasformazione di CoMbescure, curve a tangenti paralleli e, da un'altra, il caso delle coppie di curve situate su una sviluppabile. Lo studio di tale configurazione $\dot{e}$ stato fatto da BompIañ in un bellissimo lavoro inserito negli "Scritti matematici, offerti a BerzoLari [9].

Per i riferimenti del second'ordine, avremo:

$$
\omega_{3}^{1}+A \omega_{0}^{3}=\omega_{3}^{2}+B \omega_{0}^{3}=\omega_{2}^{3}+C \omega_{0}^{3}=\omega_{2}^{0}+F \omega_{0}^{3}=0, \omega_{0}^{0}+\omega_{2}^{2}+G \omega_{0}^{3}=0 .
$$

Si può prendere $A=0, B=C=-1, F=0$.

La scelta $A=F=0$ vuol dire che $A_{0} A_{3} A_{2}$ è il piano osculatore a $\Gamma_{0}$ in $A_{0} \Theta$ $A_{1} A_{2} A_{3}$ ̀े quello a $\Gamma_{1}$ in $A_{1}$, oppure che $A_{2}$ è l'intersezione della tangente a $\Gamma_{0}$ col piano osculatore a $\Gamma_{1}$ e reciprocamente. Abbiamo escluso $i$ casi $B=0$, $C=0$. Uno di essi significa che il piano osculatore ad una delle curve passa per il punto generico dell' altra. Se fosse simultaneamente $B=C=0$, si arrebbe il caso delle trastormate asintotiche - lo studio proiettivo-differenziale della quale è stato fatto, anni fa, per uno di noi. La scelta fatta a come conseguenze $\pi_{2}^{1}=\pi_{1}^{i}=\pi_{0}^{0}=\pi_{3}^{0}=0$. Essendo $\Sigma \pi_{i}^{i}=0$, tutti i parametri secondari risultano zero danque il riferimento proiettivo, determinato. Ne segue $d \omega_{0}^{3} \equiv 0$ ciò che significa che $\omega_{0}^{3}$ è invariante differenziale della configurazione; esso sarè notato per $d s$. $G$ risulta invariante del secondo ordine e sarà notato per $x$. Le conseguenze menzionate mettono in evidenza 4 invarianti del terz' ordine e si avrà

$$
\omega_{0}^{0}=\tau_{1} d s, \quad \omega_{1}^{1}=\tau_{2} d s, \quad \omega_{2}^{1}=\tau_{3} d s, \quad \omega_{3}^{0}=\tau_{4} d s
$$

e le formule di Frminet:

$$
\begin{array}{ll}
\dot{A_{0}}=\tau_{1} A_{0}+A_{3} & \dot{A}_{2}=\tau_{3} A_{1}+\left(x-\tau_{1}\right) A_{2}+A_{3} \\
\dot{A_{1}}=-\tau_{2} A_{1}+A_{2} & \dot{A}_{3}=\tau_{4} A_{0}+A_{2}+\left(\tau_{2}-x\right) A_{3} .
\end{array}
$$


Le condizioni d'immobilità saranno

$$
\begin{aligned}
\frac{\dot{x}^{0}+\tau_{1} x^{0}+\tau_{4} x^{3}}{x^{0}} & =\frac{\dot{x}^{1}-\tau_{2} x^{1}+\tau_{3} x^{2}}{x^{1}}=\frac{\dot{x}^{2}+x^{1}+\left(x-\tau_{1}\right) x^{2}+x^{3}}{x^{2}}= \\
& =\frac{\dot{x}^{3}+x^{0}+x^{2}+\left(\tau_{2}-x\right) x^{3}}{x^{3}}
\end{aligned}
$$

Interpretazioni geomelriche.

In ciò che riguarda il riferimento, rimane a caratterizzare il punto unitario. Essendo questo punto, del second'ordine della configurazione, ci conviene di considerare il regolo osculatore alla rigata $A_{0} A_{1}$ lungo questa generatrice. In coordinate locali $x^{0}, x^{1}, x^{2}, x^{3}$, l'equazione di questo regolo è

$$
2 x^{0} x^{2}-2 x^{1} x^{3}+\left(x^{2}\right)^{2}-2 x x^{2} x^{3}-\left(x^{3}\right)^{2}=0 .
$$

Le generatrici del secondo regolo di questa quadrica in $A_{0}$ e $A_{1}$ sono rispettivamente

$$
x^{2}=x^{3}+2 x^{1}=0, \quad x^{3}=x^{2}+2 x^{0}=0 .
$$

Esse segano $A_{1} A_{3}$, rispettivamente, $A_{0} A_{2}$ nei punti $V_{13}, V_{02}$. I punti unitari $U_{1}, U_{02}$ degli spigoli $A_{1} A_{3}, A_{0} A_{2}$ sono determinati dai birapporti

$$
\left[\begin{array}{llll}
A_{1}, & A_{3} ; & V_{13}, & U_{13}
\end{array}\right]=\left[\begin{array}{llll}
A_{0}, & A_{2} ; & V_{02}, & U_{02}
\end{array}\right]=-2 .
$$

Gli stessi punti $U_{13}, U_{02}$ possono essere determinati considerando le rette caratteristiche dei piani $A_{0} A_{2} A_{2}, A_{0} A_{1} A_{3}$. Le equazioni di queste rette sono

$$
x^{\mathrm{s}} \equiv x^{0}+x=0, x^{2}=x^{1}+x^{3}=0
$$

La quadrica pre-osculatrice alla rigata $A_{0} A_{1}$ passante per lo spigolo $A_{\mathbf{2}} A_{3}$ ha l'equazione

$$
x^{0} x^{2}-x^{1} x^{3}=0
$$

La retta $U_{02} U_{18}$ sega questa quadrica in due punti uno di essi essendo appunto il punto unitario del riferimento.

Nello stesso scopo, si potrebbe fare uso della "superficie principale" della coppia di curve, luogo dei vertici dei coni quadrati contenenti gli intorni del second'ordine delle curve $\Gamma_{0}, \Gamma_{1}$, superficie considerata da Halphen, Berzolari e Bompiani, la cui equazione è

$$
x^{0}\left(x^{2}\right)^{3}-x^{1}\left(x^{3}\right)^{3}=0
$$


Per trovare un' interpretazione dell' invariante differenziale, basta considerare la quaterna di piani passanti per $A_{0} A_{1}: \alpha_{2} \equiv A_{0} A_{1} A_{3}, \quad \alpha_{3} \equiv A_{0} A_{1} A_{2}$, $\pi_{0}$ e $\pi_{1}$ passanti anche per $A_{0}+d A_{0}+\frac{1}{2} d^{2} A_{0}$ rispettivamente, per $A_{1}+d A_{1}+$ $+\frac{1}{2} d^{2} A_{1}$ e si avrà

$$
d s= \pm 2 \sqrt{\text { parte pr. }\left[\alpha_{2}, \alpha_{3} ; \pi_{0}, \pi_{1}\right]}
$$

Lo spigolo $A_{2} A_{3}$, intersezione dei piani osculatrici a $\Gamma_{0}$ in $A_{0}$ e a $\Gamma_{1}$ in $A_{1}$ incontra la quadrica osculatrice alla rigata generata da $A_{0} A_{1}$ lungo questa generatrice, nei punti

$$
\left(x^{2}\right)^{2}-2 x x^{2} x^{3}-\left(x^{3}\right)^{2}=0
$$

punti che forniscono l'interpretazione dell'invariante $x$. Si può notare che l'annullare di $x$ significa ohe i punti $A_{2}, A_{3}$ sono inversi rispetto la quadrica considerata. Altre interpretazioni di $x$ si ottengono considerando $i$ piani polari di $U_{02}, U_{13}$ rispetto la stessa quadrica.

La tangente in $A_{3}$ alla curva descritta da questo punto, ha le equazioni

$$
x^{1}=x^{0}-\tau_{4} x^{2}=0 .
$$

L'intersezione di questa tangente con $A_{0} A_{2}$ fornisce I'interpretazione dell'invariante $\tau_{4}$. Analogamente, si trova l'interpretazione di $\tau_{3}$. 亡̀ da notare la relazione

$$
\tau_{4}=\left(A_{1}, \dot{A_{1}}, \ddot{A_{1}}, \ddot{A_{1}}\right)
$$

la quale mostra che $\tau_{4}=0$ significa che $\Gamma_{1}$ è una curva piana; analogamente $\tau_{3}=0$ significa che $\Gamma_{0}$ è piana.

Per trovare interpretazioni geometriche per $\tau_{1}, \tau_{2}$, basta considerare $i$ punti fleenodali, su $A_{0} A_{1}$, della superficie rigata generata da $A_{0} A_{1}$. Questi punti $F_{i}\left(1, \rho_{i}, 0,0\right)$, determinati con $\rho_{i}$ radici dell' equazione:

$$
\tau_{1} \rho^{2}+\left[\dot{x}+x\left(\tau_{2}-\tau_{1}\right)+\tau_{3}-\tau_{4}\right] \rho+\tau_{2}=0 .
$$

forniscono le interpretazioni cercate.

Notiamo che:

$\tau_{2}=0$ significa che $\Gamma_{0}$ è fleonodale della rigata $A_{0} A_{1} ;$

$\tau_{1}=0$ significa che $\Gamma_{1}$ è flecnodale della stessa rigata;

$\dot{x}+x\left(\tau_{2}-\tau_{1}\right)+\tau_{3}-\tau_{4}=0$ significa che $\mathrm{i}$ punti flecondali sono coniugati rispetto alla coppia $A_{0} A_{1}$;

in fine, se $\tau_{1}=\tau_{2}=\dot{x}+\tau_{3}-\tau_{4}=0$, la superficie $A_{0} A_{1} \grave{e}$ una quadrica. 
Osservazione. - Altre interpretazioni degli invarianti della configurazione possono ricavarsi dalla considerazione di certi complessi lineari.

Il complesso lineare contenente 3 tangenti consecutive della curva $\Gamma_{0}$ e 2 della curva $\Gamma_{1}$ ha l' equazione

$$
p_{01}+\tau_{3} p_{23}=0
$$

il complesso lineare ottenuto scambiando $\Gamma_{0}$ e $\Gamma_{1}$ tra loro ha l'equazione

$$
p_{01}+\tau_{4} p_{23}=0
$$

Questi complessi sono speciali se $\Gamma_{0}$, rispettivamente $\Gamma_{1}$, sono curve piane.

I due complessi coincidono se, e solo se, $\tau_{3}=\tau_{4}$.

In fine, tale complesso è stazionario se, di più,

$$
\frac{\dot{\tau}_{3}}{2 \tau_{3}}+\tau_{1}-\tau_{2}=0
$$

Dunque la corrispondenza tra $\Gamma_{0}$ e $\Gamma_{1}$ con $\tau_{3}-\tau_{4}=\frac{\dot{\tau}_{3}}{2 \tau_{3}}+\tau_{1}-\tau_{2}=0$ ò la più generale corrispondenza puntuale tra le curve appartenenti ad un complesso lineare.

\section{Applicazioni.}

Come abbiamo accennato al principio, dalla geometria delle coppie di curve si possono dedurre quelle della banda rigata e della superficie rigata.

10. Sia $\Gamma^{\circ}$ la curva della banda rigata considerata e $A_{0} A_{1}$ la sua retta. Facciamo coincidere $\Gamma_{1}$ con una delle curve flecnodali della rigata descritta da $A_{0} A_{1}$ ciò che da $\tau_{1}=0$. Dunque una banda rigata ammette un invariante differenziale e quattro invarianti finiti.

$\dot{E}$ facile vedere che se, tra questi invarianti, ha luogo la relazione

$$
2 \tau_{2}-x=0
$$

oppure se, nel caso $2 \tau_{2}-x \neq 0$, ha $\operatorname{luogo} \frac{d}{d s} \log \left(2 \tau_{2}-x\right)-\frac{\tau_{4}}{2 \tau_{2}-x}+\tau_{1}+$ $+\tau_{2}=0$, la banda è conica ciò che vuol dire che la sviluppabile generata dal piano $A_{0} A_{1} A_{3}$ è un cono.

$2^{0}$. Si ottiene la geometria della superficie rigata generata da $A_{0} A_{1}$, considerando $\Gamma_{0}$ e $\Gamma_{1}$ flecnodali di questa superficie. Si ha dunque 
$\tau_{2}=\tau_{1}=0$. Le formole di Frener saranno dunque

$$
\dot{A_{0}}=A_{3}, \quad \dot{A_{1}}=A_{2}, \quad \dot{A_{2}}=\tau_{3} A_{1}+x A_{2}+A_{3}, \quad \dot{A}_{3}=\tau_{4} A_{0}+A_{2}-x A_{3} .
$$

Nel caso particolare, quando $\tau_{3}$, o $\tau_{4}$, è nullo si ottiene la geometria delle superficie rigate con una o le ambedue flecnodali curve piane. Le formole di FRENET delle rigate a linee flecnodali piane e distinte sono semplicissime :

$$
\dot{A}_{0}=A_{3}, \quad \dot{A}_{1}=A_{2}, \quad \dot{A}_{2}=x A_{2}+A_{3}, \quad \dot{A}_{3}=A_{2}-x A_{3} .
$$

Lo studio di questa importante classe di superficie è stato eseguito magistralmente da 0 . Markr [10].

6. Coppie di curve nell' $S_{3}$ affine. - La configurazione delle curve $\Gamma_{0}, \Gamma_{1}$ sarà rapportata a triedri $A I_{1} I_{2} I_{3}$ con: $A$ punto generico di $\Gamma_{0}$ e $A+I_{1}=M$, punto generico di $\Gamma_{1}$. Escludendo, per momento, il caso delle coppie a tangenti incidenti, il metodo di CARTAN ci conduce alle formule di Frener:

$$
\dot{A}=I_{3}, \quad \dot{I}_{1}=I_{2}-I_{3}, \quad \dot{I}_{2}=x_{1} I_{1}+x_{2} I_{2}+x_{3} I_{3}, \quad \dot{I}_{3}=x_{4} I_{1}+x_{5} I_{2}-x_{2} I_{3} .
$$

La configurazione ha un invariante differenziale $\omega_{0}^{3}=d s$ del primo ordine e 5 invarianti finiti, $x_{1}, \ldots, x_{5}$.

Lo spigolo $A I_{2}$ del riferimento ̀̀ la parellela, per $A$, alla tangente in $M$ a $\Gamma_{1}$, mentre $A I_{3}$ è la tangente in $A$ a $\Gamma_{0}$. Il piano unitario per $A I_{1}$ è il piano asintotico della superficie rigata generata da $A I_{1}$.

Interpretazioni geometriche degli invarianti della configurazione risultano dalle considerazioni dei seguenti enti geometrici:

Il piano osculatore $\Gamma_{0}$ in $A$ ha, in coordinate locali, l'equazione

$$
x_{5} x^{1}-x_{4} x^{3}=0
$$

Quello osculatore a $\Gamma_{1}$ in $M$,

$$
x_{1} x^{3}-x_{3} x^{1}+x_{3} x^{0}=0 .
$$

La retta caratteristica del piano $A I_{1} I_{2}$ \& descritta dal punto $P \equiv M+$ $+\lambda\left(I_{2}+x_{3} I_{1}\right)$, mentre il suo punto caratteristico è definito da $\lambda$ soddisfacente

$$
\lambda\left[\dot{x}_{3}-x_{3}\left(x_{2}+x_{3}\right)+x_{1}\right]=x_{3} .
$$


Similmente, la retta caratteristica dal piano $A I_{1} I_{3}$ è descritta dal punto $Q \equiv A+\mu\left(I_{3}-x_{5} I_{1}\right)$ e il suo punto caratteristico si ottiene per:

$$
\mu\left[x_{5}\left(1+x_{2}-x_{5}\right)-x_{4}\right]=x_{5} .
$$

La caratteristica del piano $A I_{2} I_{3}: R \equiv A+v\left(x_{4} I_{2}-x_{1} I_{3}\right)$.

La quadrica osculatrice alla superficie generata da $A I_{1}$, lungo questa generatrice, ha l'equazione

$$
2 x^{1} x^{2}+2 x^{1} x^{3}-2 x^{0} x^{2}+2 x_{2} x^{2} x^{3}-x_{3}\left(x^{2}\right)^{2}+x_{5}\left(x^{3}\right)^{2}=0
$$

avente il centro nel punto $\left[2 x_{2}+x_{3}-x_{3}, x_{2}-x_{5},-1,1\right]$; questa quadrica diventa paraboloide se $2 x_{2}+x_{3}-x_{5}=0$, quando la superficie $A I_{1}$ è a piano direttore. Il punto $(\lambda, \mu, 0,0)$ è panto flecnodale sulla generatrice $A I_{1}$ se:

$$
\lambda^{2}\left(\dot{x}_{5}+x_{4}\right)+\lambda \mu\left(x_{2}-2 \dot{x}_{5}+x_{1}-x_{4}\right)+\mu^{2} \frac{d}{d s}\left(x_{5}-x_{2}-x_{3}\right)=0 .
$$

Ne segue che $\Gamma_{0}$ è flecnodale sulla rigata $A I_{1}$ se

$$
\dot{x}_{5}+x_{4}=0 ; \Gamma_{1} \text { è flecnodale se } x_{5}-x_{2}-x_{3}=c \stackrel{t e}{=} \text {. }
$$

Come nel caso proiettivo, dalla geometria della coppia di curve, si possono dedurre quelle della banda o della superficie rigata.

\section{La corrispondenza di Combescure.}

Nel caso delle coppie a tangenti paralleli, le formole di FRENET sono:

$$
\dot{A}=I_{3}, \quad \dot{I}_{1}=x I_{3}, \quad \dot{I}_{2}=\tau I_{1}+\rho I_{3}, \dot{I}_{3}=I_{2}
$$

dove $x$, $\tau$ e $\rho$ sono invarianti d'ordine 1,3 e 4 rispettivamente. Si può notare che $-\frac{1}{x}$ è l'ascisse del punto cuspidale della sviluppabile $A I_{1}$ mentre $\tau=-\left[I_{3}, \dot{I}_{3}, \ddot{I}_{3}\right]$ il quale rappresenta dunque il rapporto dell'arco affine di $\Gamma_{0}$ per $\left(\omega_{0}^{3}\right)^{3}$.

\section{BIBLIOGRAFIA}

[1] GHgorghigv GH. et Popa I., Géométrie differentielle projective des variétés de cônes, «C, R. Acad. Sci. Paris", T. 251-1960, p. 1208-1210, 1268-1269.

[2] - - Sur une variété uni-dimensionnelle non holonome de A. MruLwR, Anal. Soi. Univ. Iasi $*$, T. VI-1960, p. 59.75 . 
[3] - , Asupra unor varietati neolonome unidimensionale, "Stndii si Cerc. Acad. R. P. R. Iasi». seria Matematica, T. XJ-1960 p. 333.336.

[4] LANE E. P., Ruled surfaces with generators in one-to-one correspondance, "Trans. Amer. Math. Soc, , T. 25-1923, p. 281.296.

[5] CRnangä I., Sur une fanille de transformations entre denx surfaces réglées, "Bul. Polit. Iasi ^, 'T. II-1947, p. 35-45.

[6] IVLev E.T., Coppie di superficie rigate nello spazio proiettivo tridimensionale, in $l$. russa. "Rendiconti del Colloquio di Mat. e Meco." Università di Tomsk U.R.S.S., 1960, p. $50 \cdot 51$.

[7] Mentrí P., Sur les complexes engendrés par une congruence linéaire non speciale, C. R. Acad. Sci. Paris », T. 191-1930, p. 644-646.

[8] BaRner M., Zur projektiven Differentialgeometrie der Kurvenpaare, Math. Zeitsehr.», T. 56.1952 p. $409 \cdot 442$.

[9] Bompiani E. . Sulle curve sghembe, Scritti matematiei offerti a Lurgr BenzoLARr, 1986, p. 515-552.

[10] Maxer O., Su. les surfaces réglées à lignes flecnodales planes, Ann Sci. Univ. Jassy", T. $15-1927$, p. $25-55$. 